\title{
The Study and Simulation of Connectivity Based Routing Protocols in Ad Hoc Networks YUAN Peiyan
}

\author{
School of Physical \&Information Engineering, Henan Normal University \\ +86 0373 3326151,Xinxiang,Henan,P.R.China,453007 \\ peiyan@htu.cn
}

\begin{abstract}
Connectivity is one of the essential properties of Ad Hoc networks. It is very important to keep the whole system connective for improving the throughput of the networks. Mainly based on the method of the probability, the most literatures focus on the critical transmission range (CTR) or the averaged minimum node degree in which the Ad Hoc networks could be kconnectivity. Firstly, this paper summarizes the present process on the connectivity and simulates the four routing protocols with the energy model in the different environments such as node density, pause time and mobility velocity. Secondly, with the energy constrained, it is clearly shown that the math's models of the related works are not co insistent with the experimental results.Finaly, this paper makes a prospect for the future research areas based on the simulation results.
\end{abstract}

\section{Keywords}

Connectivity, Energy Model, Percolation Theory, Ad Hoc Networks, Network Simulation, Routing Protocol

\section{INTRODUCTION}

A mobile Ad Hoc network (MANET) is a collection of wireless mobile nodes communicating with each other using multi-hop wireless links without any existing network infrastructure or centralized administration. Each node in the network also acts as a router, forwarding data packets for other nodes. How to choice the appropriate routing between nodes is the key problem for Ad Hoc networks.

The classical routing protocols such as one distance vector routing protocol DSDV and three on-demand routing protocols AODV, DSR and TORA are all shortest routing. DSDV is a table-driven protocol based on the classical Bellman-Ford mechanism. The improvements made to Bellman-Ford algorithm include freedom from loops in the routing table. Every mobile node in the network maintains a routing table in which all of the possible destinations within the network and the number of hops to each destination are recorded. While AODV, DSR and TORA all share the on-demand behavior in that they initiate routing activity only in the presence

Permission to make digital or hard copies of all or part of this work for personal or classroom use is granted without fee provided that copies are not made or distributed for profit or commercial advantage and that copies bear this notice and the full citation on the first page. To copy otherwise, to republish, to post on servers or to redistribute to lists, requires prior specific permission and/or a fee. Copyright (9) 2008 ICST 978-1-59593-984-5 Copyright @ 2008 ICST 978-1-59593-984-5
DOI 10.4108/ICST.MOBILWARE2008.2894 of data packets in need of a route, many of their routing mechanisms are different. AODV uses a table-driven routing framework and destination sequence numbers, DSR uses a source routing, whereas TORA uses a link reversal routing mechanism. Commonly, the latter three have a less routing load and the former has a less end-to-end delay.

It's our main method to study routing protocols with simulation in different condition constrained and metrics. The current condition constrained are mainly focusing on the wireless radio model, mobility model ,energy model and the QoS factors such as throughput, delay, jitter, loss ratio and also the external characteristics such as routing load or routing time. However, using the inner property connectivity as evaluated metric, to our knowledge, the related works taken it into account are very few.

Connectivity is one of the essential properties of Ad Hoc networks. It is very important to keep the whole system connective for the improvement of the networks' throughput. Based on the small world phenomenon ${ }^{[1 \sim 8]}$, diagram theory and the continuum percolation theory ${ }^{\left[{ }^{2} 19\right]}$, the current research mainly investigate the mobile nodes in what kind of critical transmission range (CTR) ${ }^{[20 \sim 25]}$ or in what minimum degree on average $^{[25 \sim 30]}$, the network topology is $k$ connectivity. Where, the related works suppose the mobile nodes obey the Poisson distribution and make the $n$ dimension space as the study background. When supposed the network topology is an unidirectional diagram $G(V, E), V$ is the set of nodes and $E$ is the set of edges( $v i, v j), v i, v j \in V$,for the random two nodes $v i, v j$, if there is a path between them, then $G$ is 1 connective, if there is $k$ paths, it is $k$ connective. Commonly, when $r \geq \mathrm{c} 1 \times \sqrt{(\ln n+c 2) / \pi n}$ or node degree $d \geq \mathrm{c} 3 \times \log (n)$, the topology is connective. For $r, d$ and $\lambda_{c}$,the open area is very difficulty to express them exactly when $N \geq 2, k \geq 2$. Where, the node degree $d$ means the neighbor numbers of a node,r means the transmission range,c1 and c2 are the const. Obviously, it is just opponent for the above two methods, for the CTR problem, each node $r$ is fixed and $d$ is various,on the contrary, in minimum node degree problem, the $r$ is various and $d$ is fixed. The essential reason behind them is the mobile characteristic of the Ad Hoc networks, which makes the differences of the node density which impacts on the connectivity of networks in reverse.

The related works educe some constrained conditions of the $k$ connectivity, however, they do not consider the energy model, that is, all of them suppose the active nodes in entire simulation phase are constant. However, the portable communication devices in Ad Hoc networks are untethered, batteries operated and have limited energy, so the network is an energy-constrained system. It is in appropriative to study connectivity without taking into 
account the energy model. Based on the energy model, this paper simulates the relative performance of the four routing protocols on connectivity and finds that the math's models of the related works are not co insistent with the experimental results with the energy constrained.

The remainder of this paper is organized as follows. Section 2 presents the idea of small world phenomenon. Section 3 describes the continuum percolation theory. In section 4, the CTR problem is discussed. Section 5 analysis the minimum node degree, section 6 simulates the connectivity of the Ad Hoc networks. Concluding remarks and future research areas are made in Section 7.

\section{Small World Phenomenon}

The Small-World Phenomenon is very common in social network, if, roughly speaking, any two individuals in the network are likely to be connected through a short sequence of intermediate acquaintances. This has long been the subject of anecdotal observation and folklore.Often we meet a stranger and discover that we have an acquaintance in common. It has since grown into a significant area of study in the social sciences, in large part through a series of striking experiments conducted by Stanley Milgram and his co-workers in the 1960's ${ }^{[1 \sim 3]}$. Recent work has suggested that the phenomenon is pervasive in networks arising in nature and technology, and a fundamental ingredient in the structural evolution of the World Wide Web ${ }^{[4 \sim 6]}$.

Milgram's basic small-world experiment remains one of the most compelling ways to think about the problem. The goal of the experiment was to find short chains of acquaintances linking pairs of people in the United States who did not know one another. In a typical instance of the experiment, a source person in A would be given a letter to deliver to a target person in $\mathrm{B}$. The source would initially be told basic information about the target, including his address and occupation, the source would then be instructed to send the letter to someone who knew on a first-name basis in an effort to transmit the letter to the target as efficaciously as possible. Anyone subsequently receiving the letter would be given the same instructions, and the chain of communication would continue until the target was reached. Over many trials, the average number of intermediate steps in a successful chain was found to lie between five and six, a quantity that has since entered popular culture as the "six degrees of separation" principle ${ }^{[7]}$.

Kleinberg J provides a grid model to describe the small world phenomenon using the occupied theory and analysis the averaged forwarding times of a decentralized algorithm ${ }^{[8]}$. In this model, the lattice distance between two nodes to be the number of "lattice steps" separating them. A directed edge from $u$ has endpoint $v$ with probability proportional to $d(u, v)^{-r}$. It gives the mean forwarding times of the algorithm. If $0 \leqslant r<2, t \geqq a_{r} n^{(2-r) / 3}$,if $r>2, t \geqq \alpha_{r} n^{(\mathrm{r}-2) /(\mathrm{r}-1)}$, where, $t$ denotes the averaged forwarding times, $n$ means the numbers of nodes, $\alpha_{r}$ is a constant, depending on $p, q, r$, but independent of $n$.At the same time, when $r=2, p=q=1$, the upper bound of $t$ is $\alpha_{2}\left(\log _{n}\right)^{2}$; when $\delta=(2-r) / 3$, $\lambda=\left(2^{8-r} q p^{2}\right)^{-1}$,the lower bound of $t$ is $(1 / 4) \lambda n^{\delta}$. The conclusion of [8] indicates that when long-range connections are generated uniformly at random, the grid model describes a world in which short chains exist but individuals, faced with a disorienting array of social contacts, are unable to find them.

\section{Percolation Theory}

Similarity as the small world phenomenon, percolation theory ${ }^{[9 \sim 19]}$ is formed in the long society practice. Percolation as a mathematical theory was introduced by Broadbent and Hammersley, as a stochastic way of modeling the flow of a fluid or gas through a porous medium of small channels which may or may not let gas or fluid pass. It is one of the simplest models exhibiting a phase transition, and the occurrence of a critical phenomenon is central to the appeal of percolation. Having truly applied origins, percolation has been used to model the fingering and spreading of oil in water, to estimate whether one can build nondefective integrated circuits, to model the spread of infections and forest fires. From a mathematical view, the percolation theory is attractive because it exhibits relations between probabilistic and algebraic topological properties of graphs. To make the mathematical construction of such a system of channels, take a graph $G$ (which originally was taken as $\mathrm{Z}^{d}$ ), with vertex set $V$ and edge set $E$, and make all the edges independently open (or passable) with probability $p$ or closed (or blocked) with probability 1- $p$. Write $P p$ for the corresponding probability measure on the set of configurations of open and closed edges that model is called bond percolation. There is an analogous model, called site percolation, in which all edges are assumed being passable, but the vertices are independently open or closed with probability $p$ or 1-p, respectively. An open path is then a path along which all vertices are open. The most important property of the percolation model is that it exhibits a phase transition, i.e. there exists a threshold value $p c \in[0,1]$, such that the global behavior of the system is substantially different in the two regions $p<p c$ and $p>p c$. When $p<p c$ the open cluster of the origin is $P p$-a.s. finite, hence all the clusters are also finite, at that time, the network system is named in sub-critical regime. On the other hand, for $p>p c$ there is a strictly positive $P p$-probability that the cluster of the origin is infinite and the system is named in sup-critical regime. As shown in figure $1^{[13]}$ :

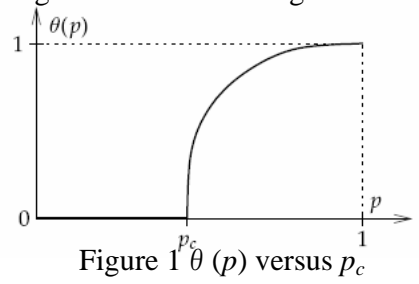

Where:

$$
\begin{aligned}
& \theta(p):=P p\{\mathbf{0} \leftrightarrow \infty\}=P p\{|C(\mathbf{0})|=\infty\} \\
& p c:=p c(G)=\sup \{p: \theta(p)=0\}
\end{aligned}
$$

H. KESTEN proved that the key parameter of bond -percolation just equal to $1 / 2$ in two dimension space ${ }^{[14]}$. The construction of 
the incipient infinite cluster was done by Kesten in two dimensions ${ }^{[15]}$, and a similar construction was performed recently in high dimension by Van der Hofstad and Jarai ${ }^{[16]}$.The result shows that in two dimensions, the random walk on the incipient infinite cluster is sub-diffusive, i.e. the mean square displacement after $n$ steps behaves as $n^{1-\varepsilon}$ for some $\varepsilon>0$.

When modified the grid model mentioned above and permitted the lattice to move arbitrary, the percolation phenomenon occurs still. The corresponding definition, due to Benjamini and Schramm ${ }^{[10]}$, is then the following: If $N$ is as above the number of infinite open clusters,

$p u:=\inf \{p: P p(N=1)=1\} \geqslant p c$

The main question is then to characterize graphs on which

$0<p c<p u<1$.Peres proved that for $p \in(p c, p u)$,there are $P p$ a.s. infinitely many infinite cluster, while for every $p \in(p u, 1)$ there is only one ${ }^{[17]}$. It is very important because which denied the guess that new infinite component could appear when $p$ is increasing. Except the above models,Sapoval puts forward a Gradient Percolation which depends on the location of $p^{[18]}$, Hammersley and Welsh makes a First passage percolation depended on time ${ }^{[19]}$ and others such as contact percolation ,invasion percolation etc.

The related works mainly used the occupied theory as their research method, in which the numbers of $n$ balls are put in $c$ lattices independently and how to put the balls into the lattices is decided by the property of the balls. The aim of the occupied theory is to make sure of the probability distribution of the variables when making a limitation of the numbers of $n$ and $c$.The occupied theory can be used to analyze the connectivity of Ad Hoc networks, by partition the zone $R$ into the numbers of $n$ small and same size areas, we can confirm the probability of one lattice holding one node at least. At the same time, the related works supposed that the numbers of $n$ nodes in one area obey the Poisson distribution with the density of $\lambda$ in two dimension space. If the distance of two nodes is less than $r$, they will be connected each other. When $\lambda>\lambda_{c}$, there would be one or more infinite components with high propability.However, if there is only one infinite component, the network would not be connected, because there may be many nodes which do not belong to it, leading to the topology partited, so the connectivity of the network topology is related to the portion of the nodes which belong to the infinite component to the nodes of all, and this portion is related to the percolation probability in reverse. Though the explicit expression of the key percolation probability has not been confirmed at present, the continuum percolation theory is still adapt to study the connectivity of Ad Hoc networks because of the model of the former consistent to the model of the latter. From the critical transmission range and minimum node degree, the below two sections illuminate the applications of the percolation theory in Ad Hoc networks.

\section{Critical Transmission Range}

One of the important problems to study connectivity is how to ascertain the critical transmission range for each node, that is, suppose every node is isomorphic and has the same transmission power, how large must the transmission range $r$ be to sure that the resulting network system be connected. Because the low-cost wireless communication components can not adjust the transmission range dynamically in wireless sensor networks, what we can do is to set the transmission range of each node just same and the only way to reduce the power consumption and improve the system capacity is to make the network connective and the transmission range as possible as minimal. Because the longest edge in MST $^{[20]}$ is just the CTR, from the probability distribution of which, the probability distribution of the CTR can be educed. The random geometry diagram theory is the most suitable way to solve the CTR problem in theory. For the dense Ad Hoc networks, the random geometry diagram theory is suitable, in which the density of node is infinite with the numbers of nodes increasing in a finite space. However,it is not suitable in reality because the density of node can not be infinite for improving the capacity of whole system. When a node is in transmission status, its neighbors must be silent, if the density is infinite, many nodes would be silent, and thus the whole system capacity is down. From the result of literature[21],when the numbers of nodes is infinite,the capacity that each node can be available is zero.

P. Gupta and P. R. Kumar firstly study the problem of $\mathrm{CTR}^{[22]}$.If $n$ nodes are located randomly, uniformly i.i.d.,in a disc of unit area and each node transmits at a power level so as to cover an area of $\pi r^{2}=(\log n+c(n)) / n$, then the resulting network is asymptotically connected with probability one if and only if $\mathrm{c}(n)$ $\rightarrow \infty$. They considered the question in a static topology and did not analyze the impact of different mobility models on CTR and the question of $k$ connectivity in static topology.

Paolo Santi study the one connectivity of CTR in a certain mobility model and prove that $r=\mathrm{c} \sqrt{(\ln n) / \pi n}^{[23]}$. They also investigate the case of random waypoint mobility and the $r=(p+0.521405 / v) / p \sqrt{(\ln n) / \pi n}$, where $p$ means the pause time and $v$ denotes the mobility velocity. However, they do not consider the $k$ connectivity in certain mobility model.

The above related works mainly study the question in dense networks. They did not research the problem in sparse networks. P. Santi and D.M. Blough study the one connectivity problem both in dense networks and in sparse networks ${ }^{[24]}$. IF $r \times$ $n \in \Theta(L \log L)$,then the system is 1 connective, if $r \times$ $n \in \mathrm{O}(L)$,then the system is not connective in $L$ dimension space, where $L$ equals to $1,2,3$. When $L$ equals to 2 or $3, k \geqslant k_{d}$ and $r=r(L)>>1$, the system is asymptotical one connective, where $k_{d}=2^{\mathrm{d}} d^{d} / 2, d=2,3$. They didn't study the $k(k \geqslant 2)$ connectivity in $L$ dimension space and not take into account the question in mobility environment. 


\section{Minimum Node Averaged Degree}

Discovering the relationship between the minimum node average degree and the connectivity is another important method to study the question. Supposing the nodes obey the uniform distribution, C. Bettstetter ${ }^{[26 \sim 27]}$ reviews the one connectivity problem in mobile model. The results are:

$$
\begin{aligned}
& \text { If } 0 \leqslant \underline{r} \leqslant 1-\underline{r}, \\
& \mu_{p}(\mathbf{x})=n \underline{r}_{0}{ }^{2} \\
& \text { If } 1-\underline{r}_{0}<\underline{r} \leqslant 1 \text {, } \\
& \mu_{p}(\mathrm{x})=n / \pi\left(\underline{r}_{0}^{2} \arccos \left(\underline{r}^{2}+\underline{r}_{0}{ }^{2}-1\right) / 2 \underline{r} \underline{r}_{0}+\arccos \left(\underline{r}^{2}-\right.\right. \\
& \left.\left.\underline{r}^{2}+1\right) / 2 \underline{r}-\mathrm{s} / 2\right) \\
& \text { If } 0 \leqslant \underline{r} \leqslant 1-\underline{r}_{0}, \\
& \mu_{m}(\mathrm{x})=n\left(-2 \underline{r}_{0}^{2} \underline{r}^{2}+2 \underline{r}_{0}^{2}-\underline{r}_{0}^{4}\right) \\
& \text { If } 1-\underline{r}_{0}<\underline{r} \leqslant 1 \text {, } \\
& \mu_{m}(\mathrm{x})=n / 4 \pi\left[4 \underline{r}_{0}^{2}\left(2 \underline{r}^{2}+\underline{r}_{0}^{2}-2\right) \arcsin \left(\underline{r}^{2}+\underline{r}_{0}^{2}-1\right) / 2 \underline{r}\right. \\
& \underline{r}_{0}-4 \arcsin \left(\underline{r}^{2}-\underline{r}_{0}^{2}+1\right) / 2 \underline{r}+\mathrm{s}\left(\underline{r}^{2}+5 \underline{r}_{0}^{2}-3\right)+2 \pi\left(-2 \underline{r}_{0}^{2} \underline{r}\right. \\
& \left.\left.{ }^{2}+2 \underline{r}_{0}{ }^{2}-\underline{r}_{0}{ }^{4}+1\right)\right] \\
& \text { then } \\
& \mu(\mathbf{x})=q \mu_{p}(\mathbf{x})+(1-q) \mu_{m}(\mathbf{x})
\end{aligned}
$$

where,s $=\operatorname{sqrt}\left(\left(\underline{r}+\underline{r}_{\underline{0}}+1\right)\left(-\underline{r}+\underline{r}_{\underline{0}}+1\right)\left(\underline{r}-\underline{r}_{\underline{0}}+1\right)\left(\underline{r}+\underline{r}_{\underline{0}}-1\right)\right)$, $\mu_{p}(\mathbf{x})$ and $\mu_{m}(\mathbf{x})$ denote the density function of the static nodes and dynamic nodes respectively and $\underline{r}_{\underline{0}}$ is the scaled transmission range. One of the concrete value of $\mu$ and $\underline{r}_{\underline{0}}$ is shown below.

$$
\mu \approx n \underline{r}_{0}^{2} / 3\left(\left(4-2 q+q^{2}\right)-4 / \pi \times q^{2}-3(1-q) \underline{r}_{0}^{2}\right)
$$

$\underline{r}_{0} \leq 0.3$

The literature [26] does not consider the question of the interference among the nodes and the problem of the various of transmission power.

O.Dousse studies the impact of interferences on connectivity and draws a conclusion that the upper bound of the averaged minimum node degree which keeps the network connective is $1+$ $1 / \beta \gamma$,where, $\beta$ is the ration of signal to noise, $\gamma$ is the inverse ratio of the system's gain ${ }^{[27]}$. Using the occupied theory, F. Xue and P. R. Kumar study the question in various transmission powers. If $d=0.074 \log n$, then the network is almost partited,if $d=5.1774 \log n$,it's 1 connectivity ${ }^{[28]}$. Except the above works, the literature [29] gives a relation between the averaged node degree and the routing hop count, the literature [30] studies the minimum node degree when the network is 1 connective.

\section{Simulation based on Energy Model}

From the mentioned above, the related works consider the question in an ideal condition without taking into account the impact of energy on connectivity.However,the portable communication devices in Ad Hoc networks are untethered, batteries operated and have limited energy, so the network is an energy-constrained system. The energy plays an important role in the actual application areas, it is inappropriate to study the connectivity left the energy model. Based on the conclusion of literature [23], this paper makes simulation with energy model. The simulator for evaluating routing protocols is implemented with the network simulation version 2 (ns2) and the topology is builded by the tool of setdest.The velocity and initial position of nodes is generated randomly and the nodes is placed within a $1000 \mathrm{~m} \times 1000 \mathrm{~m}$ area. The node mobility speed is between $0 \mathrm{~m} / \mathrm{s}$ and $40 \mathrm{~m} / \mathrm{s}$ generated by uniform distribution and the pause time is $0 s$ which means the node is always moving in the entire simulation period. A traffic generator named cbrgen is developed to simulate constant bit rate sources in ns2. This paper uses it to generate $6,12,24,30$ and 60 pair of UDP stream stochastically. Each CBR package size is 512 bytes and one second transmits one package. This paper has chosen this value because smaller payload sizes penalize protocols that append source routes to each data packet. The network initial connectivity is $2 / 3$. Radio propagation range for each node is $250 \mathrm{~m}$ and channel capacity is $2 M$ bit/s. The simulation altogether produces 210 kinds of stochastic topologies, each group of nodes corresponds 10 kinds and the collected data is the averaged over those 10 runs. The 210 kinds of stochastic topologies is classified into 4 simulations models.Firstly,the number of nodes is $10,20,40,50,100$ and the pause time is 0s.Secondly,the pause time is $0,50,100,150,200,250,300$ and the number is 50.Thirdly,the numbers of nodes is 100 , the pause time is just the same as the second. Fourthly, the velocity is $10 \mathrm{~m} / \mathrm{s}, 20 \mathrm{~m} / \mathrm{s}, 30 \mathrm{~m} / \mathrm{s}, 40 \mathrm{~m} / \mathrm{s}$, the numbers of nodes is 50 and the pause time is 0 s.There is a small portion of overlapping in the former three simulation models. The channel and radio model is two-ray ground reflection model. MAC layer uses IEEE 802.11's DCF, which is the basic access method used by mobiles to share the wireless channel under independent Ad Hoc configuration. It uses a RTS/CTS/DATA/ACK pattern for all unicast packets and simply sends out DATA for all broadcast packets. The access scheme is Carrier Sense Multiple Access/Collision Avoidance (CSMA/CA) with acknowledgments. Fragmentation is useful if a packet larger than the given threshold, however, this paper do not employ fragmentation in our experiments because the data packets are big enough that the additional overhead would reduce overall network throughput. Furthermore, we assume the ability of a radio to lock onto a sufficiently strong signal in the presence of interfering signals, i.e. radio capture. If the capture ratio (the minimum ratio of an arriving packet's signal strength relative to those of other colliding packets) is greater than the predefined threshold value, the arriving packet is received while other interfering packets are dropped.

\section{1 the Impact of Node Density on Connectivity}

The connectivity decreases for all routing protocols in the different node densities, as shown in Figure 2 to Figure 5.When considering the energy model, it shows clearly that the connectivity is decreasing quickly,however,the related works thought the network is 1connective because of not taking into account the impact of energy(here, this paper do not depict the one connectivity in these Figures and the below is just the same.). 
The reason which makes the different phenomenon is that the devices in Ad hoc networks are untethered, batteries operated and have limited energy, so the network is an energy-constrained system. The numbers of active nodes is decreasing because of the energy consumption with the simulation processing, which makes the conclusion of the literature [23] does not come into existence in practice, in which $\mathrm{r} \geqslant(p+0.521405 / v) / p \sqrt{(\ln n) / \pi n}$. One simple prove is shown below.

$\lim _{n \rightarrow 0} \sqrt{(\ln n) / \pi n}=\operatorname{sqrt}\left(\lim _{n \rightarrow 0} \ln (n) / \pi n\right)$
let $\mathrm{f}(\mathrm{x})=\ln (\mathrm{x}) /(\pi \mathrm{x})$

let sqrt denotes the evolution, if $\mathrm{x} \in[0, \mathrm{e}]$,then $\mathrm{f}(\mathrm{x})$ is increasing monotonicly,if $x \in[e,+\infty]$, then $f(x)$ is decreasing monotonically (see Figure 6).Supposed the minimal numbers of active nodes is 3 in topology, then when $n \rightarrow 3$,the formula 12 tends to 0.3415 (the value of $f(3)$ is 0.3415 ), when changing the value of $n$ from 3 to zero, the formula 11 tends to $-\infty$,then $r$ can be the value of bigger than zero at will.

From the analysis mentioned above, when we consider the energy model, the network is becoming more sparse than ever, we should increase the transmission power for sure of the connectivity.However, because of the constant of $p, v, n$ in one simulation experiment is constant in literature [23].This is a contradiction, so $r$ is not only related to the distribution of $p, v$ but also the distribution of $n$ 's decreasing, that is, $n$ is a variable, not a constant. At the same time, because of the decreasing of $\mathrm{f}(\mathrm{x}), r$ should be decreased with the node density increasing for sure of the connectivity of networks. According to the conclusion of it, $r$ is a constant in entire simulation phase, which makes the interference among nodes increasing and the termination of nodes quickly in reverse so that the connectivity is decreasing quickly in the beginning simulation phase and more worse with the node density increasing.

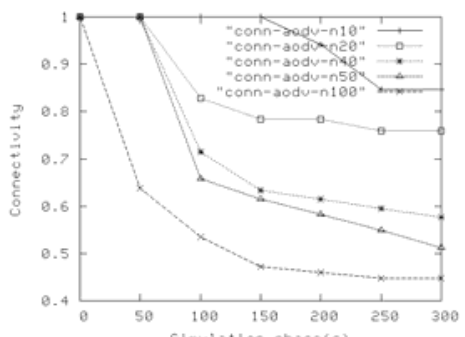

Figure2 AODV node density versus connectivity

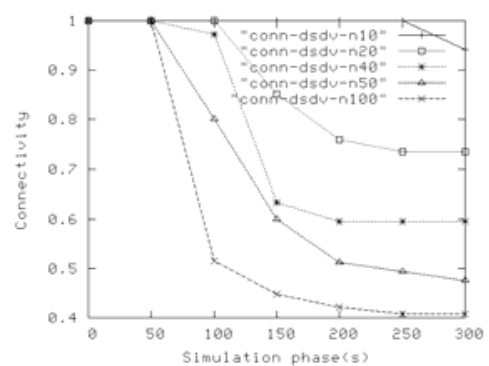

Figure3 DSDV node density versus connectivity

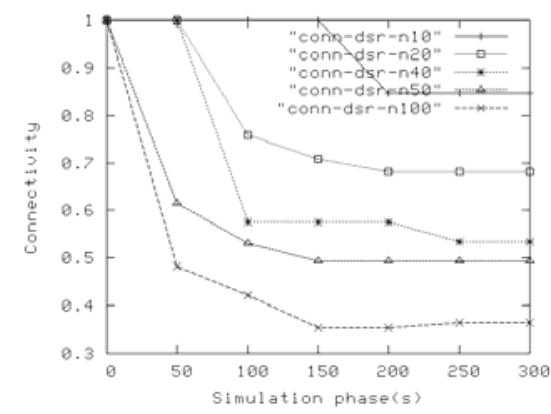

Figure4 DSR node density versus connectivity

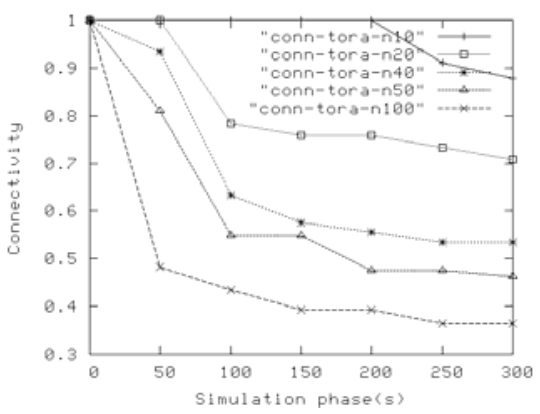

Figure5 TORA node density versus connectivity

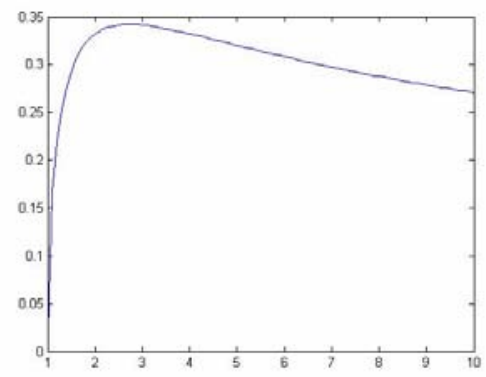

Figure 6 the image of $f(x)$

Figure 7 to Figure 11 show the connective performance of the four routing protocols in the different node densities.DSDV and AODV have the better characteristics than DSR and TORA in general.

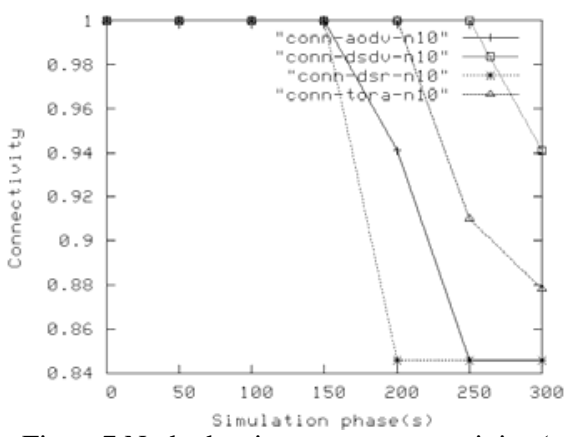

Figure7 Node density versus connectivity $(n=10)$ 


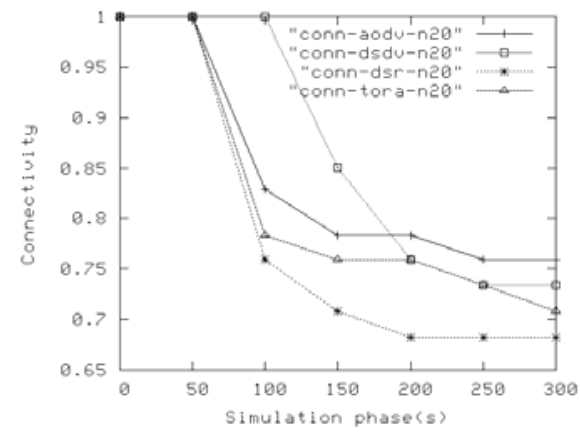

Figure8 Node density versus connectivity $(n=20)$

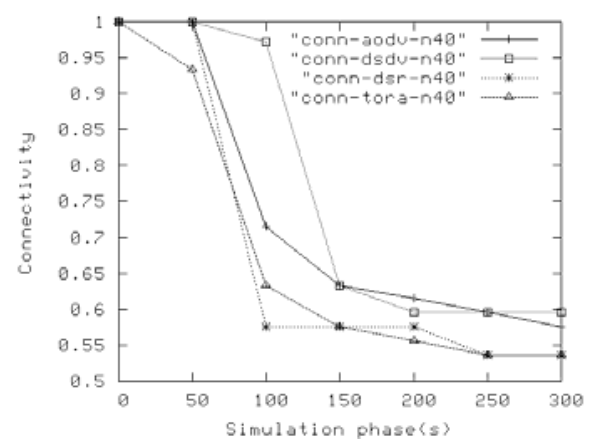

Figure9 Node density versus connectivity $(n=40)$

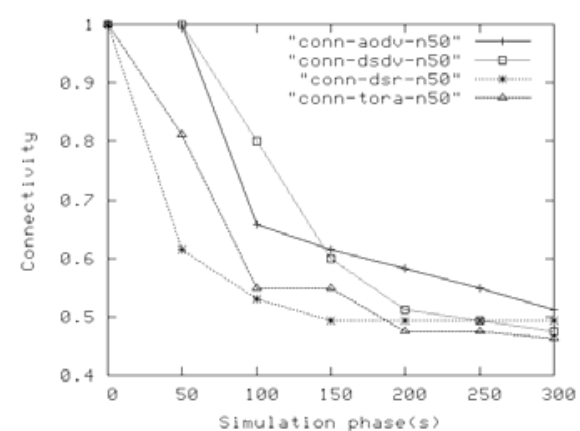

Figure10 Node density versus connectivity $(n=50)$

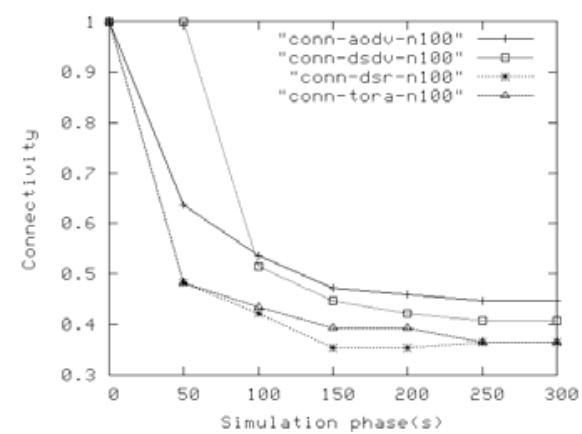

Figure11 Node density versus connectivity $(n=100)$

\section{2 the Impact of Pause Time on Connectivity}

For the four routing protocols, the connectivity of the topology is increasing with the pause time increasing, as shown from Table 1 and Table 2 in the numbers of nodes 50 and 100 respectively(P means pause time and $\mathrm{T}$ means the type of routing protocols). With the increasing of the pause time, the status of the topology is varying from dynamic to static, so the communication and interference probability is decreasing among the nodes, which, on the one hand,makes the energy consumption of nodes and the number of active nodes change slowly, on the other hand, leads to the $r$ is changing slowly too, so it is in favor of maintaining the connectivity of network.

Table 1 the numbers of death nodes in different pause time

\begin{tabular}{|c|c|c|c|c|c|c|c|}
\hline $\mathrm{T}^{\mathrm{p}}$ & 0 & 50 & 100 & 150 & 200 & 250 & 300 \\
\hline AODV & 44 & 42 & 42 & 39 & 42 & 35 & 31 \\
\hline DSDV & 46 & 47 & 44 & 35 & 43 & 36 & 34 \\
\hline DSR & 45 & 48 & 46 & 39 & 45 & 41 & 28 \\
\hline TORA & 47 & 44 & 46 & 42 & 45 & 41 & 38 \\
\hline
\end{tabular}

Table 2 the numbers of death nodes in different pause time

\begin{tabular}{|c|c|c|c|c|c|c|c|}
\hline $\mathrm{T}^{\mathrm{P}}$ & 0 & 50 & 100 & 150 & 200 & 250 & 300 \\
\hline AODV & 90 & 89 & 87 & 88 & 80 & 89 & 76 \\
\hline DSDV & 93 & 93 & 88 & 92 & 89 & 92 & 71 \\
\hline DSR & 98 & 94 & 91 & 90 & 87 & 94 & 79 \\
\hline TORA & 98 & 96 & 94 & 94 & 95 & 93 & 88 \\
\hline
\end{tabular}

At the same pause time, AODV and DSDV show the better performance than the other two, but in a longer pause time, the better is AODV and DSR. DSDV has a better energy consumption balance in dynamic topology than static, but the DSR is the other way round. In all circumstances, TORA shows a worst performance. Because TORA uses a link reversal routing mechanism, when one node detects one of its neighbors is loss, it makes its sequence number be the biggest among its neighbors, which make the traffic bypass it. With the increasing of the similar nodes, the connectivity is down quickly.

\section{3 the Impact of Velocity on Connectivity}

The varying of the connectivity of the four routing protocols is shown from table 3 in different velocities. As a whole, the connectivity is worst in velocity $30 \mathrm{~m} / \mathrm{s}$ and biggest in $40 \mathrm{~m} / \mathrm{s}$ (except AODV).From the related works, the connectivity is closely related to the active nodes which are affected by the status of the energy consumption. With the increasing of the velocity, the probability of the nodes becoming neighbors is increasing too, which on the one hand, makes the connectivity better, on the other hand, makes the nodes consume much more energy, which will be impact the numbers of active nodes and the connectivity in reverse.Obviously,it is a pair of contradiction and the process form $20 \mathrm{~m} / \mathrm{s}$ to $30 \mathrm{~m} / \mathrm{s}$ may be a key phase. We should pay more attention to it when we study the energy-constrained Ad Hoc networks. 
At the same velocity,AODV and DSDV have a better characteristic than DSR and TORA,which is different from the performance at the same pause time. Integrated with the section 6.2, DSDV is fit for the varying of velocity and has a sensitive to the changing of pause time.

Table 3 the numbers of death nodes in different velocities

\begin{tabular}{|c|c|c|c|c|}
\hline $\mathrm{T}^{\mathrm{V}}$ & 10 & 20 & 30 & 40 \\
\hline AODV & 41 & 33 & 41 & 44 \\
\hline DSDV & 44 & 40 & 43 & 46 \\
\hline DSR & 44 & 37 & 45 & 45 \\
\hline TORA & 44 & 44 & 47 & 47 \\
\hline
\end{tabular}

\section{Conclusion and Future Works}

Connectivity is one of the essential properties of Ad Hoc networks. It is very important to keep the whole system connective for the improvement of the networks' throughput. This paper reviews it from the four areas. The related works study the one connectivity in a static topology and suppose the nodes are i.i.d., most of them do not consider the impact of mobility model and energy model on the $k$ connectivity. The math's models of the related works are not co insistent with the experimental results when considering the energy model (See section six).How to models and analysis the question in math's is our present works.

\section{Acknowledgement:}

The work is supported by the National Natural Science Foundation Grant 90304018 of China.

\section{REFERENCES}

[1] S. Milgram. The small world problem [J]. Psychology Today, 1967, (22): 61-67.

[2] J. Travers, S. Milgram. An experimental study of the small world problem [J]. Sociometry,1969, 32(4):425-443.

[3] C. Korte and S. Milgram. Acquaintance networks between racial groups: Application of the small world method[J].Personality and Social Psych.,1978, 15(2):101118.

[4] H. Kautz, B. Selman, M. Shah. Referral Web: Combining Social Networks and Collaborative Filtering[J]. Communications of the ACM, 1997, 40(3):63-65.

[5] D.Watts and S.Strogatz.Collective dynamics of small-world networks [J].Nature, 1998, 393(6684):440 442.

[6] R. Albert, H. Jeong, A.-L.Barabasi. The diameter of the World Wide Web [J]. Nature, 1999, 401:130-131.

[7] J. Guare. Six Degrees of Separation: A Play [M].Vintage Books, New York, 1990.

[8] J.Kleinberg.The Small-World Phenomenon: An Algorithmic Perspective [J]. Nature, 2000, 406:67-98.
[9] S.R.BROADBENT, J.M.HAMMERSLEY.Percolation processes, I and II[C]. Crystals and. Mazes. Proc.Cambridge Philos. Soc, 1957, 629-645.

[10] I. BENJAMINI AND O. SCHRAMM.Percolation beyond Zd, many questions and a few answers [J].Electron. Comm. Probab, 1996, 1, (8):71-82.

[11] G.GRIMMETT.Percolation [M].seconded, Springer-Verlag, Berlin, 1999.

[12] L. Booth, J. Bruck, M. Franceschetti, and R. Meester.Covering Algorithms, Continuum Percolation and the Geometry of Wireless Networks[J].Annals of Applied Probability, 2003, 13(2):722-741.

[13] VincentBeffara.PercolationTheory[EB/OL].http://www.ump a.ens-lyon.fr/ vbeffara/files/Enc-Perco.pdf ,July 122005.

[14] H. KESTEN.The critical probability of bond percolation on the square lattice equals 1/2[J]. Comm.Math. Phys, 1980, 74: 41-59.

[15] H. KESTEN.The incipient infinite cluster in twodimensional percolation [J]. Probab. Theory Related Fields, 1986, 73:369-394.

[16] R. VAN DER HOFSTAD AND A. A. JÁRAI.The incipient infinite cluster for high-dimensional un-oriented percolation [J]. J. Statist. Phys.2004, 114: 625-663.

[17] Y.PERES.Probability on trees: an introductory climb, in Lectures on probability theory and statistics [C]. Saint-Flour, vol. 1717 of Lecture Notes in Math. Berlin: Springer, 1999, 193-280.

[18] B.SAPOVAL, M.ROSSO, J.GOUYET.The fractal nature of a diffusion front and the relation to percolation [J]. J. Phys. Lett, 1985, 46:146-156.

[19] J.M.HAMMERSLEY, D.J.A.WELSH. Firstpassagepercolation, subadditive processes, stochastic networks, and generalized renewal theory[C]. Calif, in Proc. Internat. Res. Semin, Statist.Lab, Univ.California, Berkeley, New York: Springer-Verlag, 1965, 61-110.

[20] M. Penrose. A strong law for the longest edge of the minimal spanning tree [J]. Ann. Probab. 1999, 27 (1):246-260.

[21] P.Gupta, P.R.Kumar.The Capacity of Wireless Networks [J].IEEE Transactions on Information Theory, 2000, 46(2):388-404.

[22] P. Gupta, P. R. Kumar. Critical Power for Asymptotic Connectivity in Wireless Networks[C]. Stochastic Analysis, Control, Optimization and Applications, Boston: Birkhäuser, 1998,547-566.

[23] P. Santi. The Critical Transmitting Range for Connectivity in Mobile Ad Hoc Networks [J]. IEEE Trans. on Mobile Computing, 2005, 4(3):310-317.

[24] P.Santi, D.M.Blough.The Critical Transmitting Range for Connectivity in Sparse Wireless Ad hoc Networks [J]. IEEE Trans. on Mobile Computing, 2003, 2:25-39.

[25] P.-J. Wan, C.-W. Yi.Asymptotic Critical Transmission Radius and Critical Neighbor Number for k-Connectivity in Wireless Ad Hoc Networks[C]. ACM MobiHoc'04, Tokyo, Japan: IEEE Computer Society Press, 2004:1-8. 
[26] C. Bettstetter.Topology properties of ad hoc networks with random waypoint mobility[C]. ACM MobiHoc, Annapolis.MD.USA: IEEE Computer Society Press, 2003.

[27] O. Dousse, F. Baccelli, P. Thiran. Impact of interferences on connectivity in ad-hoc networks[C]. IEEE INFOCOM, San Francisco, CA: IEEE Computer Society Press, 2003, 1724-1733.

[28] F.Xue, P.R.Kumar.The Number of Neighbors needed for Connectivity of Wireless Networks [J]. Wireless Networks, 2004, 10:169-181.
[29] R.Hekmat, P.VanMieghem.Degree Distribution and Hop count in Wireless Ad-hoc Networks[C].IEEE ICON'03, Sydney, Australia: IEEE Computer Society Press, 2003, 603609.

[30] G. Ferrari, O. K. Tonguz.Minimum Number of Neighbors for Fully Connected Uniform Ad Hoc Wireless Networks[C]. IEEE ICC'04, Paris, France: IEEE Computer Society Press, 2004. 